Research Article

\title{
On New Modifications of Some Perturbation Procedures
}

\author{
A. I. Ismail $\mathbb{D}^{1,2}$ \\ ${ }^{1}$ Mechanical Engineering Department, College of Engineering and Islamic Architecture, Umm Al-Qura University, Makkah, \\ Saudi Arabia \\ ${ }^{2}$ Mathematics Department, Faculty of Science, Tanta University, Tanta, P.O. Box 31527, Egypt \\ Correspondence should be addressed to A. I. Ismail; aiismail@uqu.edu.sa
}

Received 29 October 2020; Revised 25 December 2020; Accepted 28 January 2021; Published 9 February 2021

Academic Editor: Jorge E. Macias-Diaz

Copyright (C) 2021 A. I. Ismail. This is an open access article distributed under the Creative Commons Attribution License, which permits unrestricted use, distribution, and reproduction in any medium, provided the original work is properly cited.

\begin{abstract}
In this paper, we present new modifications for some perturbation procedures used in mathematics, physics, astronomy, and engineering. These modifications will help us to solve the previous problems in different sciences under new conditions. As problems, we have, for example, the rotary rigid body problem, the gyroscopic problem, the pendulum motion problem, and other ones. These problems will be solved in a new manner different from the previous treatments. We solve some of the previous problems in the presence of new conditions, new analysis, and new domains. We let complementary conditions of such studied previously. We solve these problems by applying the large parameter technique used by assuming a large parameter which inversely proportional to a small quantity. For example, in rigid body dynamic problems, we take such quantity to be one of the components of the angular velocity vector in the initial instant of the rotary body about a fixed point. The domain of our solutions will be depending on the choice of a large parameter. The problem of slow (weak) oscillations is considered. So, we obtain slow motions of the bodies instead of fast motions and find the solutions of the problem in present new conditions on both of center of gravity, moments of inertia, and the angular velocity vector or one of these parameters of the body. This study is important for aerospace engineering, gyroscopic motions, satellite motion which has the correspondence of inertia moments, antennas, and navigations.
\end{abstract}

\section{Introduction}

In [1], the variation of parameters method is applied to solve the general nonhomogenous linear differential equation of the second order. The author solved the homogenous equation and constructed a particular solution depending on its solutions. He got the first and second differentiation of the particular solutions, substituted them into the considered equation, and performed some investigated steps, and he found the required general solution.

We note that this is an exact solution for a linear differential equation and not an approximated one and so is not the small parameter technique or the large parameter one useful for this problem. Such techniques are useful only for approximated problems consisting of nonlinear differential equations containing a small parameter [2] or a large one $[3,4]$. In the following sections, we modify the well-known previous method in terms of the large parameter for solving some systems for some perturbed problems $[5,6]$ containing quasi-linear systems. This modification gives us the chance to study the considered problems in new conditions and new domains of solutions.

\section{The Method of Averaging}

We consider the following examples for illustrating this method, and we will find the periodic solutions of each case:

2.1. Van Der Pol's Technique. This technique is considered in previous works for finding the approximated periodic solutions as power series in terms of a small parameter defined for each problem. In our work, we aim to find new periodic solutions in terms of a large parameter instead of the small one of the equation

$$
\frac{\mathrm{d}^{2} u}{\mathrm{~d} t^{2}}+\omega_{0}^{2} u=\varepsilon^{-1}\left[\left(1-u^{2}\right) \frac{\mathrm{d} u}{\mathrm{~d} t}+K \lambda \cos \lambda t\right]
$$


where $\varepsilon$ is imposed to be large and $\lambda=O\left(\varepsilon^{-1}\right)$ is the movement frequency which is assumed to be small to be different from the natural frequency $\omega_{0}$. By taking the solution to (1), we have assumed the following form:

$$
u(t)=a_{1}(t) \cos \lambda t+a_{2}(t) \sin \lambda t,
$$

where $a_{1}(t)$ and $a_{2}(t)$ are taken to be slow functions that vary with time as well as $\left(\mathrm{d} a_{i} / \mathrm{d} t\right)=0\left(\varepsilon^{-1}\right)$ and $\left(d^{2} a_{i} / d t^{2}\right)=0\left(\varepsilon^{-2}\right)$. By differentiating (2) twice, we obtain

$\ddot{u}=-\lambda^{2} a_{1} \cos \lambda t-\lambda^{2} a_{2} \sin \lambda t-2 \dot{a}_{1} \lambda \sin \lambda t+2 \dot{a}_{2} \lambda \cos \lambda t+\ddot{a}_{1} \cos \lambda t+\ddot{a}_{2} \sin \lambda t, \quad\left(\dot{u} \equiv \frac{\mathrm{d} u}{\mathrm{~d} t}\right)$.

Using equations (1)-(3), we obtain

$$
\begin{aligned}
2 \dot{a}_{1}+a_{2}\left(\lambda-\frac{\omega_{0}^{2}}{\lambda}\right)-\varepsilon^{-1} a_{1}(1-\rho) & =0, \\
2 \dot{a}_{2}-a_{1}\left(\lambda-\frac{\omega_{0}^{2}}{\lambda}\right)-\varepsilon^{-1} a_{2}(1-\rho) & =\varepsilon^{-1} K, \\
\rho & =0.25 a^{2}=0.25\left(a_{1}^{2}+a_{2}^{2}\right) .
\end{aligned}
$$

Periodic solutions of (1) match solutions:

$$
\begin{gathered}
2 \sigma a_{20}-a_{10}\left(1-\rho_{0}\right)=0, \\
-2 \sigma a_{10}-a_{20}\left(1-\rho_{0}\right)=K,
\end{gathered}
$$

where $\sigma=\lambda-\varepsilon \omega_{0}$.

By adding squares (6) and (7) and using (5), we obtain the solution of the frequency equation:

$$
\rho_{0}\left[4 \sigma^{2}+\left(1-\rho_{0}\right)^{2}\right]=0.25 K^{2} \text {. }
$$

2.2. The Krylov-Bogoliubov Technique. This technique considered through the weak nonlinear equation of the second order in terms of the small parameter was defined in the problem [7-11]. Here, we consider applying the large parameter $\varepsilon$. Let us consider the following equation:

$$
\frac{\mathrm{d}^{2} u}{\mathrm{~d} t^{2}}+\omega_{0}^{2} u=\varepsilon^{-1} f\left(u, \frac{\mathrm{d} u}{\mathrm{~d} t}\right)
$$

At $\varepsilon \longrightarrow \infty$, the solution of (9) can be written as follows:

$$
u=a \cos \left(\omega_{0} t+\theta\right)
$$

where $a$ and $\theta$ are constants. To determine the approximated solution of (9) for the large parameter $\varepsilon$, Krylov and Bogoliubov hypothesized that the solution is still given by
(10) but with the time change $a$ and $\theta$, and it is subjected to the condition:

$$
\begin{gathered}
\frac{\mathrm{d} u}{\mathrm{~d} t}=-a \omega_{0} \sin \phi, \\
\phi=\omega_{0} t+\theta .
\end{gathered}
$$

By differentiation (10) w. r. t.t, we obtain

$$
\frac{\mathrm{d} u}{\mathrm{~d} t}=-a \omega_{0} \sin \phi+\frac{\mathrm{d} a}{\mathrm{~d} t} \cos \phi-a \frac{\mathrm{d} \theta}{\mathrm{d} t} \sin \phi
$$

So,

$$
\frac{\mathrm{d} a}{\mathrm{~d} t} \cos \phi-a \frac{\mathrm{d} \theta}{\mathrm{d} t} \sin \phi=0
$$

By differentiation (11) w. r. t.t, we obtain

$$
\frac{\mathrm{d}^{2} u}{\mathrm{~d} t^{2}}=-a \omega_{0}^{2} \cos \phi-\omega_{0} \frac{\mathrm{d} a}{\mathrm{~d} t} \sin \phi-a \omega_{0} \frac{\mathrm{d} \theta}{\mathrm{d} t} \cos \phi .
$$

Substituting this expression into (9) and using (10), we obtain

$$
\begin{aligned}
\omega_{0} \frac{\mathrm{d} a}{\mathrm{~d} t} \sin \phi+a \omega_{0} \frac{\mathrm{d} \theta}{\mathrm{d} t} \cos \phi & =-\varepsilon^{-1} f, \\
f & =f\left[a \cos \phi,-a \omega_{0} \sin \phi\right] .
\end{aligned}
$$

Solving (13) and (15) for $(\mathrm{d} a / \mathrm{d} t)$ and $(\mathrm{d} \theta / \mathrm{d} t)$, we obtain that

$$
\begin{aligned}
\omega_{0} \frac{\mathrm{d} a}{\mathrm{~d} t} & =-\varepsilon^{-1} \sin \phi f, \\
a \omega_{0} \frac{\mathrm{d} \theta}{\mathrm{d} t} & =-\varepsilon^{-1} \cos \phi f .
\end{aligned}
$$

By integrating (16) into the period $[t, t+T]$, where $a$ and $\theta$ can be taken as constants on the right-hand side, we obtain 


$$
\begin{array}{r}
2 \omega_{0} \frac{\mathrm{d} a}{\mathrm{~d} t}=-\varepsilon^{-1} f_{1}(a), \\
2 a \omega_{0} \frac{\mathrm{d} \theta}{\mathrm{d} t}=-\varepsilon^{-1} g_{1}(a),
\end{array}
$$

where

$$
\begin{aligned}
& \pi f_{1}(a)=\int_{0}^{2 \pi} \sin \phi f \mathrm{~d} \phi, \\
& \pi g_{1}(a)=\int_{0}^{2 \pi} \cos \phi f \mathrm{~d} \phi .
\end{aligned}
$$

We also note that $f_{1}$ and $g_{1}$ are coefficients of the Fourier series expansion of the function $f$.

As an example, let us consider the Duffing equation.

In this example, we modify the Duffing equation in terms of the large parameter to be

$$
\frac{\mathrm{d}^{2} u}{\mathrm{~d} t^{2}}+\omega_{o}^{2} u=-\varepsilon^{-1} u^{3}
$$

where

$$
f(u, \dot{u})=-u^{3}
$$

So,

$$
\begin{aligned}
& f_{1}(a)=0, \\
& g_{1}(a)=-0.75 a^{3} .
\end{aligned}
$$

Thus, from (17), it is obtained

$$
\omega_{o} \theta=0.375 \varepsilon^{-1} a^{2} t+\omega_{o} \theta_{o}, \quad a=\text { const. }
$$

Therefore, the first approximation is

$$
u=a \cos \omega_{o}\left[1+\left(0.375 \varepsilon^{-1} a^{2} / \omega_{o}^{2}\right)\right] t+o\left(\varepsilon^{-2}\right) .
$$

For the second example, consider the Van der Pol oscillator:

$$
\frac{\mathrm{d}^{2} u}{\mathrm{~d} t^{2}}+\omega_{o}^{2} u=f(u, \dot{u})
$$

where

$$
f(u, \dot{u})=\left(1-u^{2}\right) \frac{\mathrm{d} u}{\mathrm{~d} t} .
$$

In this case,

$$
\begin{array}{r}
f_{1}=-\omega_{o} a\left(1-0.25 a^{2}\right), \\
g_{1}=0 .
\end{array}
$$

Hence and from (17), it follows that $(\mathrm{d} a / \mathrm{d} t)$ is obtained in terms of the large parameter, so

$$
\begin{aligned}
\frac{\mathrm{d} a}{\mathrm{~d} t} & =0.5 \varepsilon^{-1} a\left(1-0.25 a^{2}\right), \\
\theta & =\theta_{o}=\text { const. }
\end{aligned}
$$

By integrating the first equation into (27), it becomes clear that

$$
a^{2}=\frac{4}{\left[1+\left(\left(4 / a_{o}\right)-1\right) e^{\varepsilon t}\right]}
$$

2.3. The Generalized Method of Averaging. This method was applied for obtaining the periodic solutions of the differential equations applying the small parameter technique which was defined for each case of the motion [12-14]. In our work, we use the large parameter method for obtaining new solutions dependent on the large parameter $\varepsilon$. Let us consider (10) and (11) as the conversion from $u$ and $(\mathrm{d} u / \mathrm{d} t)$ to $a$ and $\phi$ in terms of the large parameter, so

$$
\begin{aligned}
\omega_{o} \frac{\mathrm{d} a}{\mathrm{~d} t} & =-\varepsilon^{-1} \sin \phi f, \\
a \omega_{o} \frac{\mathrm{d} \phi}{\mathrm{d} t} & =a \omega_{o}^{2}-\varepsilon^{-1} \cos \phi f,
\end{aligned}
$$

where $\phi$ is called the rapid rotation phase. Let us take the conversions from $(a, \phi)$ to $(\bar{a}, \bar{\phi})$ such that

$$
\begin{aligned}
& a=\bar{a}+\varepsilon^{-1} a_{1}(\bar{a}, \bar{\phi})+\varepsilon^{-2} a_{2}(\bar{a}, \bar{\phi})+\ldots, \\
& \phi=\bar{\phi}+\varepsilon^{-1} \phi_{1}(\bar{a}, \bar{\phi})+\varepsilon^{-2} \phi_{2}(\bar{a}, \bar{\phi})+\ldots
\end{aligned}
$$
form:

Using (30), we find that system (29) takes the following

$$
\begin{aligned}
& \frac{\mathrm{d} \bar{a}}{\mathrm{~d} t}=\varepsilon^{-1} A_{1}(\bar{a})+\varepsilon^{-2} A_{2}(\bar{a})+\ldots, \\
& \frac{\mathrm{d} \bar{\phi}}{\mathrm{d} t}=\omega_{o}+\varepsilon^{-1} B_{1}(\bar{a})+\varepsilon^{-2} B_{2}(\bar{a})+\ldots,
\end{aligned}
$$

where $A_{i}$ and $B_{i}$ do not depend on $\bar{\phi}$, for each $i=1,2$.

Using (29)-(31), we obtain

$$
\begin{aligned}
& \omega_{o} \frac{\partial a_{n}}{\partial \bar{\phi}}+A_{n}=F_{n}(\bar{a}, \bar{\phi}), \\
& \omega_{o} \frac{\partial \phi_{n}}{\partial \bar{\phi}}+B_{n}=G_{n}(\bar{a}, \bar{\phi}) .
\end{aligned}
$$

Generally, $F_{n}$ and $G_{n}$ contain short periodic terms (referred to as superscript $s$ ) and long periodic terms (referred to by superscript $\ell$ ).

We choose $A_{n}$ and $B_{n}$ such that it is equal to the long periodic terms, so

$$
\begin{aligned}
& A_{n}=F_{n}^{\ell}, \\
& B_{n}=G_{n}^{\ell},
\end{aligned}
$$

then 


$$
\begin{aligned}
& \omega_{o} \frac{\partial a_{n}}{\partial \bar{\phi}}=F_{n}^{s}, \\
& \omega_{o} \frac{\partial \phi_{n}}{\partial \bar{\phi}}=G_{n}^{s},
\end{aligned}
$$

which can be solved, respectively, in $a_{n}$ and $\phi_{n}$.

As an example, consider the Van der Pol oscillator (24) in which

$$
f(u, \dot{u})=\left(1-u^{2}\right) \dot{u}, \quad \omega_{o}=1 .
$$

In this case, equation (29) in terms of the large parameter becomes:

$$
\begin{aligned}
& \frac{\mathrm{d} a}{\mathrm{~d} t}=0.125 \varepsilon^{-1}\left[a\left(4-a^{2}\right)-4 a \cos 2 \phi+a^{3} \cos 4 \phi\right], \\
& \frac{\mathrm{d} \phi}{\mathrm{d} t}=1+0.125 \varepsilon^{-1}\left[2\left(2-a^{2}\right) \sin 2 \phi-a^{2} \sin 4 \phi\right] .
\end{aligned}
$$

By substituting (30) and (31) into (36) and equating the coefficients of similar powers of $\varepsilon$ we obtain the following. Order $\varepsilon^{-1}$ :

$\frac{\partial a_{1}}{\partial \bar{\phi}}+A_{1}=0.125 \bar{a}\left(4-\bar{a}^{2}\right)-0.5 \bar{a} \cos 2 \bar{\phi}+0.125 \bar{a}^{3} \cos 4 \bar{\phi}$, $\frac{\partial \phi_{1}}{\partial \bar{\phi}}+B_{1}=0.25\left(2-\bar{a}^{2}\right) \sin 2 \bar{\phi}-0.125 \bar{a}^{2} \sin 4 \bar{\phi}$.

Order $\varepsilon^{-2}$ :

$$
\begin{aligned}
\frac{\partial a_{2}}{\partial \bar{\phi}}+A_{2}= & -\frac{\partial a_{1}}{\partial \bar{a}} A_{1}-\frac{\partial a_{1}}{\partial \bar{a}} B_{1}+0.125 a_{1}\left[4-3 \bar{a}^{2}-4 \cos 2 \bar{\phi}+3 \bar{a}^{2} \cos 4 \bar{\phi}\right] \\
& +0.5 \bar{a} \phi\left[2 \sin 2 \bar{\phi}-\bar{a}^{2} \sin 4 \bar{\phi}\right], \\
\frac{\partial \phi_{2}}{\partial \bar{\phi}}+B_{2}= & -\frac{\partial \phi_{1}}{\partial \bar{a}} A_{1}-\frac{\partial \phi_{1}}{\partial \bar{\phi}} B_{1}-0.25 \bar{a} a_{1}(2 \sin 2 \bar{\phi}+\sin 4 \bar{\phi}) \\
& +0.5 \phi_{1}\left[\left(2-\bar{a}^{2}\right) \cos 2 \bar{\phi}-\bar{a}^{2} \cos 4 \bar{\phi}\right] .
\end{aligned}
$$

Equally, $A_{1}$ and $B_{1}$ by the long periodic terms on the right-hand side in (37) is obtained as

$$
A_{1}=0.125 \bar{a}\left(4-\bar{a}^{2}\right), \quad B_{1}=0 .
$$

Hence, system (37) becomes

$$
\begin{aligned}
& \frac{\partial a_{1}}{\partial \bar{\phi}}=-0.5 \bar{a} \cos 2 \bar{\phi}+0.125 \bar{a}^{3} \cos 4 \bar{\phi}, \\
& \frac{\partial \phi_{1}}{\partial \bar{\phi}}=0.25\left(2-\bar{a}^{2}\right) \sin 2 \bar{\phi}-0.125 \bar{a}^{2} \sin 4 \bar{\phi} .
\end{aligned}
$$

So, we obtain the following solutions

$$
\begin{aligned}
& a_{1}=-0.25 \bar{a} \sin 2 \bar{\phi}+0.031 \bar{a}^{3} \sin 4 \bar{\phi}, \\
& \phi_{1}=-0.125\left(2-\bar{a}^{2}\right) \cos 2 \bar{\phi}+0.031 \bar{a}^{2} \cos 4 \bar{\phi} .
\end{aligned}
$$

By using (39) and (41), the relation (38) becomes:

$$
\begin{aligned}
& \frac{\partial a_{2}}{\partial \bar{\phi}}+A_{2}=\text { short }- \text { period terms } \\
& \frac{\partial \phi_{2}}{\partial \bar{\phi}}+B_{2}=-0.125+0.188 \bar{a}^{2}-0.043 \bar{a}^{4}
\end{aligned}
$$

$$
+ \text { short - period terms. }
$$

Equally, $A_{2}$ and $B_{2}$ by the long periodic terms on the right-hand side in (42) is obtained as

$$
\begin{array}{r}
A_{2}=0, \\
B_{2}=-0.125+0.188 \bar{a}^{2}-0.043 \bar{a}^{4} .
\end{array}
$$

Therefore, the solution up to the second approximation $\left(1 / \varepsilon^{2}\right)$ becomes

$$
u=a \cos \phi
$$

where 


$$
\begin{aligned}
a & =\bar{a}-0.125 \varepsilon^{-1} \bar{a}\left[\sin 2 \bar{\phi}-0.125 \bar{a}^{2} \sin 4 \bar{\phi}\right]+o\left(\varepsilon^{-2}\right), \\
\phi & =\bar{\phi}-0.125 \varepsilon^{-1}\left[\left(2-\bar{a}^{2}\right) \cos 2 \bar{\phi}-0.25 \bar{a}^{2} \cos 4 \bar{\phi}\right]+o\left(\varepsilon^{-2}\right), \\
\frac{\mathrm{d} \bar{a}}{\mathrm{~d} t} & =0.125 \varepsilon^{-1} \bar{a}\left(4-\bar{a}^{2}\right)+o\left(\varepsilon^{-2}\right) \\
\frac{\mathrm{d} \bar{\phi}}{\mathrm{d} t} & =1-0.125 \varepsilon^{-2}\left[1-1.5 \bar{a}^{2}+0.344 \bar{a}^{4}\right]+o\left(\varepsilon^{-3}\right) .
\end{aligned}
$$

\section{Struble's Technique}

Struble developed a technique for treating weak nonlinear oscillatory systems such as those governed by a second-order differential equation in terms of a small parameter [15]. We treat this equation in terms of the large parameter as follows:

$$
\ddot{u}+\omega_{0}^{2} u=\varepsilon^{-1} f(u, \dot{u}, t) .
$$

Let us consider the solution of (46) in terms of the large parameter $\varepsilon$, which takes the form

$$
u=a \cos \left(\omega_{0} t-\theta\right)+\sum_{n=1}^{N} \varepsilon^{-n} u_{n}(t)+o\left(\varepsilon^{-N-1}\right),
$$

where $a$ and $\theta$ are functions that are slowly changing with time.

As an example, we take Duffing's equation (19) and use (47) to give

$$
\begin{gathered}
{\left[2 a \omega_{0} \frac{\mathrm{d} \theta}{\mathrm{d} t}+\frac{d^{2} a}{\mathrm{~d} t^{2}}-a\left(\frac{\mathrm{d} \theta}{\mathrm{d} t}\right)^{2}\right] \cos \left(\omega_{0} t-\theta\right)+\left[-2 \omega_{0} \frac{\mathrm{d} a}{\mathrm{~d} t}+a \frac{d^{2} \theta}{\mathrm{d} t^{2}}\right.} \\
\left.+2 \frac{\mathrm{d} a}{\mathrm{~d} t} \frac{\mathrm{d} \theta}{\mathrm{d} t}\right] \sin \left(\omega_{0} t-\theta\right)+\varepsilon^{-1}\left(\frac{d^{2} u_{1}}{\mathrm{~d} t^{2}}+\omega_{0}^{2} u_{1}\right)+\varepsilon^{-2}\left(\frac{d^{2} u_{2}}{\mathrm{~d} t^{2}}+\omega_{0}^{2} u_{2}\right)+\ldots \\
=-\varepsilon^{-1} a^{3} \cos ^{3}\left(\omega_{0} t-\theta\right)-3 \varepsilon^{-2} u_{1} a^{2} \cos ^{2}\left(\omega_{0} t-\theta\right)+\ldots \\
u_{1}=0.031 \omega_{0}^{-2} a^{3} \cos 3\left(\omega_{0} t-\theta\right) .
\end{gathered}
$$

Considering the terms of order $O\left(\varepsilon^{-1}\right)$ and equality of coefficients $\cos \left(\omega_{0} t-\theta\right)$ and $\sin \left(\omega_{0} t-\theta\right)$ on both sides, we get the so-called variation equations:

$$
\begin{array}{r}
2 a \omega_{0} \frac{\mathrm{d} \theta}{\mathrm{d} t}+\frac{d^{2} a}{\mathrm{~d} t^{2}}-a\left(\frac{\mathrm{d} \theta}{\mathrm{d} t}\right)^{2}=-0.75 \varepsilon^{-1} a^{3}, \\
-2 a \omega_{0} \frac{\mathrm{d} a}{\mathrm{~d} t}+a \frac{d^{2} \theta}{\mathrm{d} t^{2}}+2 \frac{d a}{\mathrm{~d} t} \frac{d \theta}{\mathrm{d} t}=0 .
\end{array}
$$

While the perturbed equations remain, so

$$
\frac{d^{2} u_{1}}{\mathrm{~d} t^{2}}+\omega_{0}^{2} u_{1}=-0.25 a^{3} \cos 3\left(\omega_{0} t-\theta\right) .
$$

For the first order of $(1 / \mathcal{E})$, equation (49) reduces to

$$
\begin{aligned}
& \frac{\mathrm{d} a}{\mathrm{~d} t}=0, \\
& \frac{\mathrm{d} \theta}{\mathrm{d} t}=-0.375 \varepsilon^{-1}\left(\frac{a^{2}}{\omega_{0}}\right) .
\end{aligned}
$$

Hence,

$$
\begin{aligned}
& a=a_{0}, \\
& \theta=\left(-0.375 \varepsilon^{-1}\left(\frac{a_{0}^{2} t}{\omega_{0}}\right)\right)+\theta_{0},
\end{aligned}
$$

where $a_{o}$ and $\theta_{o}$ are constants. If we consider $\theta$ and $a$ are constants, the solution of (50) for the first order becomes
Hence, the solution for the first-order $O(1 / \varepsilon)$ becomes

$$
u=a \cos \left(\omega_{0} t-\theta\right)+0.031 \omega_{0}^{-2} \varepsilon^{-1} a^{3} \cos 3\left(\omega_{0} t-\theta\right),
$$

where $a$ and $\theta$ are given from equation (52).

Satisfying the solution for second order, we use (53) for calculating the term:

$$
\begin{aligned}
& -3 \varepsilon^{-2} u_{1} a^{2} \cos ^{2}\left(\omega_{0}-\theta\right)=-0.023 \omega_{0}^{-2} \varepsilon^{-2} a^{5}\left[\cos \left(\omega_{0} t-\theta\right)\right. \\
& \left.+2 \cos 3\left(\omega_{0} t-\theta\right)+\cos 5\left(\omega_{0} t-\theta\right)\right] .
\end{aligned}
$$

We note that the expression $\left(\left(d^{2} u_{1} / \mathrm{d} t^{2}\right)+\omega_{0}^{2} u_{1}\right)$ contains a term of order $O(1 / \varepsilon)$ in the following form:

$$
0.563 \omega_{0}^{-2} a^{3} \frac{\mathrm{d} \theta}{\mathrm{d} t} \cos 3\left(\omega_{0} t-\theta\right) .
$$

Now, we consider the order $O\left(1 / \varepsilon^{2}\right)$ leads us to the variation equations:

$$
\begin{aligned}
& 2 a \omega_{0} \frac{\mathrm{d} \theta}{\mathrm{d} t}+\frac{d^{2} a}{\mathrm{~d} t^{2}}-a\left(\frac{\mathrm{d} \theta}{\mathrm{d} t}\right)^{2}=-0.75 \varepsilon^{-1} a^{3}-0.023 \omega_{0}^{-2} \varepsilon^{-2} a^{5} \\
& -2 a \omega_{0} \frac{\mathrm{d} a}{\mathrm{~d} t}+a \frac{d^{2} \theta}{\mathrm{d} t^{2}}+2 \frac{\mathrm{d} a}{\mathrm{~d} t} \frac{\mathrm{d} \theta}{\mathrm{d} t}=0,
\end{aligned}
$$

and the perturbed equation is 


$$
\begin{aligned}
\frac{d^{2} u_{2}}{\mathrm{~d} t^{2}}+\omega_{0}^{2} u_{2}= & -0.023 \omega_{0}^{-2} a^{5}\left[2 \cos 3\left(\omega_{0} t-\theta\right)+\cos 5\left(\omega_{0} t-\theta\right)\right] \\
& -0.563 \omega_{0}^{-1} \varepsilon^{-1} a^{3} \frac{\mathrm{d} \theta}{\mathrm{d} t} \cos 3\left(\omega_{0} t-\theta\right)
\end{aligned}
$$

The solution of equation (57) can be obtained from the recurrence principle of (52) to obtain

$$
\begin{aligned}
& a=a_{0}, \\
& \theta=-0.375 \omega_{0}^{-1} \varepsilon^{-1} a_{0}^{2} t+0.059 \omega_{0}^{-3} \varepsilon^{-2} a_{0}^{4} t+\theta_{0}+o\left(\varepsilon^{-3}\right),
\end{aligned}
$$

where $a_{o}$ and $\theta_{o}$ are constants. Substituting by $(\mathrm{d} \theta / \mathrm{d} t)$ from (52) into (58) and solving the resulted equation, we obtain

$$
u_{2}=-0.001 \omega_{0}^{-4} a^{5}\left[21 \cos 3\left(\omega_{0} t-\theta\right)-\cos 5\left(\omega_{0} t-\theta\right)\right] .
$$

Thus, we obtain the solution up to the second approximation in the form

$$
\begin{aligned}
u= & a \cos \left(\omega t-\theta_{0}\right)+0.031 \varepsilon^{-1} a^{3} \omega_{0}^{-2}\left(1-0.656 \varepsilon^{-1} \omega_{0}^{-2} a^{2}\right) \cos 3\left(\omega t-\theta_{0}\right) \\
& +0.001 \varepsilon^{-2} a^{5} \omega_{0}^{-4} \cos 5\left(\omega t-\theta_{0}\right)+o\left(\varepsilon^{-3}\right)
\end{aligned}
$$

where

$$
\omega=\omega_{0}\left(1+0.375 \varepsilon^{-1} a^{2} \omega_{0}^{-2}-0.059 \varepsilon^{-2} a^{4} \omega_{0}^{-4}\right)+o\left(\varepsilon^{-3}\right) .
$$

The periodic time $T=(2 \pi / \omega)$ is given in the form

$$
T=2 \pi \omega_{0}^{-1}+\alpha\left(\varepsilon^{-1}\right),
$$

where the correction of the period is obtained in the form $\alpha\left(\varepsilon^{-1}\right)=2 \pi \omega_{0}^{-1}\left[-0.375 \varepsilon^{-1} a^{2} \omega_{0}^{-2}+0.199 \varepsilon^{-2} a^{4} \omega_{0}^{-4}+o\left(\varepsilon^{-3}\right)\right]$.

\section{The Krylov-Bogoliubov-Mitropolski Technique}

This technique is devoted to finding approximated periodic solutions using the small parameter as in [7-11]. In our work, we modify the approximated expansions using the large parameter to become

$$
u=a \cos \psi+\sum_{n=1}^{N} \varepsilon^{-n} u_{n}(a, \psi)+o\left(\varepsilon^{-N-1}\right),
$$

where each $u_{n}$ is a periodic function in $\psi$, and its period $2 \pi$, $a$, and $\psi$ are imposed to change with time as follows:

$$
\begin{aligned}
& \frac{\mathrm{d} a}{\mathrm{~d} t}=\sum_{n=1}^{N} \varepsilon^{-n} A_{n}(a)+o\left(\varepsilon^{-N-1}\right), \\
& \frac{\mathrm{d} \psi}{\mathrm{d} t}=\omega_{o}+\sum_{n=1}^{N} \varepsilon^{-n} \psi_{n}(a)+o\left(\varepsilon^{-N-1}\right),
\end{aligned}
$$

where the functions $u_{n}, A_{n}$, and $\psi_{n}$ are arbitrary so that equations (65) to (67) satisfy the differential equation (9). The following derivatives series are obtained:

$$
\begin{aligned}
\frac{d}{\mathrm{~d} t} & =\frac{\mathrm{d} a}{\mathrm{~d} t} \frac{\partial}{\partial a}+\frac{\mathrm{d} \psi}{\mathrm{d} t} \frac{\partial}{\partial \psi} \\
\frac{d^{2}}{\mathrm{~d} t^{2}} & =\left(\frac{\mathrm{d} a}{\mathrm{~d} t}\right)^{2} \frac{\partial^{2}}{\partial a^{2}}+\frac{d^{2} a}{\mathrm{~d} t^{2}} \frac{\partial}{\partial a}+2 \frac{\mathrm{d} a}{\mathrm{~d} t} \frac{\mathrm{d} \psi}{\mathrm{d} t} \frac{\partial^{2}}{\partial a \partial \psi}+\left(\frac{\mathrm{d} \psi}{\mathrm{d} t}\right)^{2} \frac{\partial^{2}}{\partial \psi^{2}}+\frac{d^{2} \psi}{d t^{2}} \frac{\partial}{\partial \psi} \\
\frac{d^{2} a}{\mathrm{~d} t^{2}} & =\frac{\mathrm{d} a}{\mathrm{~d} t} \sum_{n=1}^{N} \varepsilon^{-n} \frac{\mathrm{d} A_{n}}{\mathrm{~d} a}=\varepsilon^{-2} A_{1} \frac{\mathrm{d} A_{1}}{\mathrm{~d} a}+o\left(\varepsilon^{-3}\right) \\
\frac{d^{2} \psi}{\mathrm{d} t^{2}} & =\frac{\mathrm{d} a}{\mathrm{~d} t} \sum_{n=1}^{N} \varepsilon^{-n} \frac{\mathrm{d} \psi_{n}}{\mathrm{~d} a}=\varepsilon^{-2} A_{1} \frac{\mathrm{d} \psi_{1}}{\mathrm{~d} a}+o\left(\varepsilon^{-3}\right)
\end{aligned}
$$


We will visualize this method in the following example.

$$
\begin{aligned}
& \omega_{o}\left(\frac{\partial^{2} u_{1}}{\partial \psi^{2}}+u_{1}\right)=2\left(\psi_{1} a \cos \psi+A_{1} \sin \psi\right)-a^{3} \omega_{o}^{-1} \cos ^{3} \psi \\
& \omega_{o}^{2}\left(\frac{\partial^{2} u_{2}}{\partial \psi^{2}}+u_{2}\right)= {\left[a\left(2 \omega_{o} \psi_{2}+\psi_{1}^{2}\right)-A_{1} \frac{\mathrm{d} A_{1}}{\mathrm{~d} a}\right] \cos \psi+\left[2\left(\omega_{o} A_{2}+A_{1} \psi_{1}\right)\right.} \\
&\left.+a A_{1} \frac{\mathrm{d} \psi_{1}}{\mathrm{~d} a}\right] \sin \psi-3 u_{1} a^{2} \cos ^{2} \psi-2 \omega_{o}\left(\psi_{1} \frac{\partial^{2} u_{1}}{\partial \psi^{2}}+A_{1} \frac{\partial^{2} u_{1}}{\partial a \partial \psi}\right)
\end{aligned}
$$

Let $u_{1}$ be periodic, and the singular terms in the righthand side of (70) must be deleted. Since

$$
\cos ^{3} \psi=0.75(3 \cos \psi+\cos 3 \psi),
$$

then equation (70) gives

$$
\begin{aligned}
& A_{1}=0, \\
& \psi_{1}=0.375 a^{2} \omega_{o}^{-1} .
\end{aligned}
$$

The solution of equation (70) becomes

$$
u_{1}=0.031 a^{3} \omega_{o}^{-2} \cos 3 \psi
$$

Substituting (74) into (71) gives

$$
\begin{aligned}
\omega_{o}^{2}\left(\frac{\partial^{2} u_{2}}{\partial \psi^{2}}+u_{2}\right)= & \left(2 \omega_{o} \psi_{2}+0.117 a^{4} \omega_{o}^{-2}\right) a \cos \psi+2 \omega_{o} A_{2} \sin \psi \\
& +0.008 a^{5} \omega_{o}^{-2}(21 \cos 3 \psi-3 \cos 5 \psi) .
\end{aligned}
$$

Canceling the singular terms yields

$$
\begin{aligned}
& A_{2}=0, \\
& \psi_{2}=-0.059 a^{4} \omega_{o}^{-3} .
\end{aligned}
$$

$$
u_{2}=-0.001 a^{5} \omega_{o}^{-4}(21 \cos 3 \psi-\cos 5 \psi) .
$$

Therefore, the second approximated solution $u$ is given in the following form:

The solution of (75) becomes

$$
u=a \cos \psi+0.031 \varepsilon^{-1} a^{3} \omega_{0}^{-2} \cos 3 \psi-0.001 \varepsilon^{-2} a^{5} \omega_{0}^{-4}(21 \cos 3 \psi-\cos 5 \psi)+o\left(\varepsilon^{-3}\right)
$$

where

$$
\begin{aligned}
\frac{\mathrm{d} a}{\mathrm{~d} t} & =0 \text { or } a=a_{0}=\text { const. } \\
\frac{\mathrm{d} \psi}{\mathrm{d} t} & =\omega_{0}+0.375 \varepsilon^{-1} a^{2} \omega_{0}^{-1}-0.059 \varepsilon^{-2} a^{4} \omega_{0}^{-3}+o\left(\varepsilon^{-3}\right), \\
\psi & =\omega_{0}\left[1+0.375 \varepsilon^{-1} a^{2} \omega_{0}^{-2}-0.059 \varepsilon^{-2} a^{4} \omega_{0}^{-4}\right] t+\psi_{0}+o\left(\varepsilon^{-3}\right),
\end{aligned}
$$

where $\psi_{o}$ is constant.

\section{Multiple Scale Method}

This technique is considered for solving the differential equations applying the small parameter $[1,16]$. In our work, we modify this method to be suitable for the large parameter technique.

From equation (46), we put $\omega_{o}=1$, and we obtain 


$$
\ddot{u}+u=\varepsilon^{-1} f(u, \dot{u}, t) .
$$

To have accurate and regular expansion over all periods for the solution of this equation, we should be finding an approximated representation of the function $u\left(t, \varepsilon^{-1}\right)$ as shown in the form

$$
u\left(t, \varepsilon^{-1}\right)=\sum_{n=0}^{N} \varepsilon^{-n} u_{n}^{*}\left(t_{0}, \ldots, t_{N}\right)+o\left(\varepsilon^{-N-1}\right),
$$

where

$$
t_{m}=\varepsilon^{-m} t, \quad(m=0,1,2, \ldots, N) .
$$

Therefore, the total differential of (80) gives

$$
\frac{d}{\mathrm{~d} t}=\sum_{n=o}^{N} \varepsilon^{-n} \frac{\partial}{\partial t_{n}} .
$$

Substituting expansions (81) and (83) into equation (80), then equalizing the coefficients of equal powers for $\varepsilon^{-1}$ on both sides, and by solving the resulting equations, the solutions are obtained. Such solutions contain arbitrary functions in the variables $t_{1}, \ldots, t_{N}$ and will be determined with a condition $u_{o}^{*}$ and $\left(u_{n}^{*} / u_{n-1}^{*}\right)$ which are bounded to each $t_{n}$.

Now, we will apply the modified method to the following example.

5.1. Duffing Equation. Consider the Duffing equation in terms of the large parameter which takes the form

$$
\frac{d^{2} x}{\mathrm{~d} t^{2}}+\omega_{0}^{2} x+\varepsilon^{-1} x^{3}=0
$$

Taking

$$
x=\sum_{n=o}^{2} \varepsilon^{-n} x_{n}\left(t_{o}, t_{1}, t_{2}\right)+o\left(\varepsilon^{-3}\right),
$$

substituting (85) in (84), and equating the like powers of $\mathcal{\varepsilon}^{-1}$ to zero, we obtain

$$
\begin{array}{r}
D_{0}^{2} x_{0}+\omega_{0}^{2} x_{0}=0, \\
D_{0}^{2} x_{1}+\omega_{0}^{2} x_{1}+2 D_{0} D_{1} x_{0}+x_{0}^{3}=0, \\
D_{0}^{2} x_{2}+\omega_{0}^{2} x_{2}+2 D_{0} D_{1} x_{1}+\left(2 D_{0} D_{2}+D_{1}^{2}\right) x_{0}+3 x_{0}^{2} x_{1}=0,
\end{array}
$$

where $D_{n}=\left(\partial / \partial t_{n}\right)$.

The solution of equation (86) is

$$
x_{0}=A\left(t_{1}, t_{2}\right) e^{i \omega_{0} t}+\bar{A}\left(t_{1}, t_{2}\right) e^{-i \omega_{0} t},
$$

$$
\begin{aligned}
D_{0}^{2} x_{1}+\omega_{0}^{2} x_{1}= & {\left[2 i \omega_{0} D_{1} \bar{A}-3 A \bar{A}^{2}\right] e^{-i \omega_{0} t}-\left[2 i \omega_{0} D_{1} A+3 A^{2} \bar{A}\right] e^{i \omega_{0} t} } \\
& -A^{3} e^{3 i \omega_{0} t}-\bar{A}^{3} e^{-3 i \omega_{0} t} .
\end{aligned}
$$

Since $\left(x_{1} / x_{0}\right)$ is finite for each $t$, the singular terms must be neglected. So,

$$
\begin{aligned}
& 2 i \omega_{0} D_{1} A+3 A^{2} \bar{A}=0, \\
& 2 i \omega_{0} D_{1} \bar{A}-3 A \bar{A}^{2}=0,
\end{aligned}
$$

where $\bar{A}$ is the complex conjugate of $A$. Then, equation (87) becomes as follows:

thus

$$
\begin{aligned}
A & =A_{1}\left(t_{2}\right) e^{\left(3 i A_{1} \bar{A}_{1} t_{1} / 2 \omega_{0}\right)}, \\
x_{1} & =B e^{i \omega_{0} t}+\bar{B} e^{-i \omega_{0} t}+0.125 \omega_{0}^{-2}\left[A^{3} e^{3 i \omega_{0} t}+\bar{A}^{3} e^{-3 i \omega_{0} t}\right] .
\end{aligned}
$$


Using $x_{0}$ and $x_{1}$ into (88) yields

$$
\begin{aligned}
D_{0}^{2} x_{2}+\omega_{0}^{2} x_{2}= & -0.375 \omega_{0}^{-2}\left[A^{5} e^{5 i \omega_{0} t}+\bar{A}^{5} e^{-5 i \omega_{0} t}\right]+3 A^{2}\left[0.875 \omega_{0}^{-2} A^{2} \bar{A}-B\right] e^{3 i \omega_{0} t} \\
& +3 \bar{A}^{2}\left[0.875 \omega_{0}^{-2} A \bar{A}^{2}-\bar{B}\right] e^{-3 i \omega_{0} t}+Q e^{i \omega_{0} t}+\bar{Q} e^{-i \omega_{0} t},
\end{aligned}
$$

where

$$
Q=-2 i \omega_{0}\left(D_{1} B+D_{2} A\right)-3 A(A \bar{B}+2 B \bar{A})+1.875 \omega_{0}^{-2} A^{3} \bar{A}^{2} .
$$

By eliminating the singular terms, therefore $\left(x_{2} / x_{1}\right)$ will be finite for all $t$; then,

$$
\begin{aligned}
B & =0, \\
2 i \omega_{0} D_{2} A_{1} & =1.875 \omega_{0}^{-2} A_{1}^{3} \bar{A}_{1}^{2} .
\end{aligned}
$$

Therefore,

$$
A_{1}=A_{2} e^{-0.938 i A_{2}^{2} \bar{A}_{2}^{2} \omega_{0}^{-3} t_{2}} .
$$

A solution $x_{2}$ regardless of the homogeneous solution is given:

$$
x_{2}=0.016 \omega_{0}^{-4}\left[A^{5} e^{5 i \omega_{0} t}+\bar{A}^{5} e^{-5 i \omega_{0} t}-21 A \bar{A}\left(A^{3} e^{3 i \omega_{0} t}+\bar{A}^{3} e^{-3 i \omega_{0} t}\right)\right] .
$$

Finally, we get the solution $x$ in terms of the large parameter up to the second approximation of $(1 / \varepsilon)$ in the form

$$
\begin{aligned}
x= & a \cos (\omega t+\phi)+0.031 \varepsilon^{-1} a^{3} \omega_{0}^{-2}\left(1-0.656 \varepsilon^{-1} a^{2} \omega_{0}^{-2}\right) \cos 3(\omega t+\phi) \\
& +0.001 \varepsilon^{-2} a^{5} \omega_{0}^{-4} \cos 5(\omega t+\phi),
\end{aligned}
$$

where $A=0.5 a e^{i \phi}$ and $\omega=\omega_{0}+0.375 \varepsilon^{-1} a^{2} \omega_{0}^{-1}-0.059 \varepsilon^{-2}$ $a^{4} \omega_{0}^{-3}$. The obtained solution has the same period (63).

\section{Conclusion}

In this paper, we modified some of the perturbation methods that are considered ones of the innovations of mathematicians and physicists for finding approximated solutions to important problems. We cannot find a complete solution to these problems. Nayfeh, Poincare, and Krylov are among the prominent scientists in this field as they dealt with many problems in mathematics and physics which are applied in many branches, such as classical mechanics, flexible body mechanics, and many other branches. All these methods depend on a small parameter that was defined for each problem.

The study of such methods shows that the large parameter method is the easiest, most accurate, and one of the methods most used to solve many important problems efficiently. The large parameter method has an advantage over the other methods because it solves the problem in a new domain when it fails all other methods for solving the problem in such a domain. One of the most important application is when we study the slow spin motion of a rigid body in a Newtonian field of force under an external moment [4], the rotational motion of a heavy solid in a uniform gravity field [3], or the gyroscopic motions with a sufficiently small angular velocity component about the major or the minor axis of the ellipsoid of inertia. There are many applications of this technique in aerospace science, satellites, navigations, antennas, and solar collectors. This technique is also useful in all perturbed problems in physics and mechanics, for example, the perturbed pendulum motions and the perturbed mechanical systems.

\section{Data Availability}

No data were used to support the findings of the study. 


\section{Conflicts of Interest}

The authors declare that they have no conflicts of interest.

\section{References}

[1] A. H. Nayfeh, Introduction to Perturbation Techniques, pp. 360-364, WILEY-VCH Verlag GmbH \& Co. KGaA, Hoboken, NJ, USA, 2011.

[2] I. A. Arkhangel'skii, Construction of Periodic Solutions for the Euler-Poisson Equations by Means of Power Series Expansions Containing a Small Parameter, Colloquia Mathematica Societatis Janos Bolyai, Keszthely, Hungary, 1975.

[3] A. I. Ismail, "Solving a problem of rotary motion for a heavy solid using the large parameter method," Advances in Astronomy, vol. 2020, Article ID 2764867, , 2020.

[4] A. I. Ismail, "The slow spinning motion of a rigid body in Newtonian field and external torque," Advances in Astronomy, vol. 2020, Article ID 4179590, , 2020.

[5] A. P. Proskuriakov, "Periodic oscillations of a quasilinear autonomous system with two degrees of freedom," Public Money \& Management, vol. 24, no. 6, pp. 1103-1109, 1960.

[6] Z. H. A. Sartabanov and B. Z. H. Omarova, On Multi-Periodic Solutions of Quasilinear Autonomous Systems with an Operator of Differentiation on the Lyapunov's Vector Field, K. Zhubanov Aktobe Regional State University, Aktobe, Kazakhstan, 2019.

[7] N. N. Bogoliubov and Y. A. Mitropolski, Asymptotic Methods in the Theory of Non-linear Oscillations, Gordon and Breach, New York, NY, USA, 1961.

[8] A. I. Ismail, "On the application of Krylov-bogoliubovmitropolski technique for treating the motion about a fixed point of a fast spinning heavy solid," Zeitschrift für Wirtschaftsgeographie, vol. 20, pp. 205-208, 1996.

[9] A. I. Ismail, "Periodic solutions of equations of motion of a heavy solid applying Krylov-Bogoliubov-Mitropolski method," Journal of Computational and Applied Mathematics, vol. 114, no. 2, pp. 345-359, 2000.

[10] T. S. Amer, A. I. Ismail, and W. S. Amer, "Application of the krylov-bogoliubov-mitropolski technique for a rotating heavy solid under the influence of a gyrostatic moment," Journal of Aerospace Engineering, vol. 25, no. 3, pp. 421-430, 2012.

[11] T. S. Amer and I. M. Abady, "On the application of KBM method for the 3-D motion of asymmetric rigid body," Nonlinear Dynamics, vol. 89, no. 3, pp. 1591-1609, 2017.

[12] Y. A. Mitropolski, "Averaging methods in non-linear mechanics," International Journal of Non-Linear Mechanics, vol. 2, no. 1, pp. 69-96, 1967.

[13] A. I. Ismail, T. S. Amer, S. A. El Banna, and M. A. El-Ameen, "Electromagnetic gyroscopic motion," Journal of Applied Mathematics, vol. 2012, Article ID 12645, , 2012.

[14] T. S. Amer, "The rotational motion of the electromagnetic symmetric rigid body," Applied Mathematics \& Information Sciences, vol. 10, no. 4, pp. 1453-1464, 2016.

[15] F. Lakrad and M. M. Charafi, "Perturbation methods and the Melnikov functions for slowly varying oscillators," Chaos, Solitons \& Fractals, vol. 25, no. 3, pp. 675-680, 2005.

[16] P. K. Jakobsen, "Introduction to the method of multiple scales," 2016, https://arxiv.org/abs/1312.3651. 